\title{
Splitting the Fast and Slow Motions in Molecular Dynamics Simulations Based on the Change of Cold Potential Well Bottom
}

\author{
Zhijun Zheng ${ }^{\mathrm{a}} *$ and Yilong Bai ${ }^{\mathrm{a}}$ \\ ${ }^{a}$ LNM, Institute of Mechanics, Chinese Academy of Sciences, Beijing 100190, P.R. China \\ (*zjzheng@lnm.imech.ac.cn)
}

\begin{abstract}
The atomic motion is coupled by the fast and slow components due to the high frequency vibration of atoms and the low frequency deformation of atomic lattice, respectively. A two-step approximate method was presented to determine the atomic slow motion. The first step is based on the change of the location of the cold potential well bottom and the second step is based on the average of the appropriate slow velocities of the surrounding atoms. The simple tensions of one-dimensional atoms and two-dimensional atoms were performed with the full molecular dynamics simulations. The conjugate gradient method was employed to determine the corresponding location of cold potential well bottom. Results show that our two-step approximate method is appropriate to determine the atomic slow motion under the low strain rate loading. This splitting method may be helpful to develop more efficient molecular modeling methods and simulations pertinent to realistic loading conditions of materials.
\end{abstract}

Keywords: Molecular simulations; Multiple-time scale problem; Cold potential well. PACS: $33.15 . \mathrm{Vb}$

\section{INTRODUCTION}

The Molecular Dynamics (MD) method is still limited to a few nanoseconds in time and a few nanometers in size due to its physical essence. To simulate deformations under realistic applied loading rate from $10^{-6} / \mathrm{s}$ to $10^{6} / \mathrm{s}$ with molecular simulation is desired but not yet available, although some techniques, like the Multiple Time-Step (MTS) method [1-4], have been developed. Therefore, to explore how to split and combine the fast and slow motions, namely the high frequency (thermal) motion with atomic frequency of about $10^{13} / \mathrm{s}$ and the low frequency deformation of atomic lattice, is a key to this multiple-time scale problem. For this sake, we propose a splitting method in this paper.

In the MTS molecular dynamics simulations, the interatomic forces are split into the fast and slow components corresponding to the short and long range forces, respectively [1-4]. In the standard MD simulations, the forces must be recomputed at each time step [5]. Differently, in the MTS method, the fast component of force is computed after each time step and the slow component of force is computed every $n$ time steps. So it can reduce the CPU time and speedup the simulations. But the efficiency is not enough high to simulate the case of the realistic loading conditions.

The coupling effect of the high and low frequency motions of atom seriously restricts the development of the more efficient molecular modeling methods and simulations pertinent to realistic loading conditions of materials. In solid materials, most atoms locate at their local potential well formed by the surrounding atoms and their motions at the macro level remain stable and continuous until losing stability. The coupling of atomic thermal motions and mechanical deformation of materials may be weak before losing stability and it makes the splitting fast and slow motions possible. 
In the next section, we present a two-step approximate method to determine the atomic slow motion and split the atomic fast and slow motions. The validity of this method is illustrated by simulating the simple tensions of onedimensional atoms and two-dimensional atoms.

\section{CONTENTS}

The motions of atoms are complex. For the solid material under external loading, there are two motions: high frequency motion due to the thermal excitation and low frequency deformation of atomic lattice according to external loading rate. In form, the motion of atom can be split into the fast and slow motions. The velocity of atom $i$ in the $\alpha$ axis direction can be written as

$$
v_{i \alpha}(t)=v_{i \alpha}^{\mathrm{H}}(t)+v_{i \alpha}^{\mathrm{L}}(t),
$$

where the superscripts $\mathrm{H}$ and $\mathrm{L}$ correspond to the fast and slow components of atomic motion and $t$ is the time. The fast and slow components are coupled in some kind of unknown physical mechanism. It is difficult to split them due to the nonlinear effect and there are no independent control equations to determine the two components, respectively. To explore the coupling mechanism may be a key to this multiple-time scale problem and will help us to develop a new algorithm to speedup the molecular simulations. The time average method [6] is a popular one to calculate the statistical values of physical parameters, but it is not an effective method to approximately calculate the atomic slow velocity. In this section we present another approximate method for determining the atomic slow velocity.

\section{Method for Determining the Atomic Slow Velocity}

It is well known that every atom in a lattice is restrained in its local potential well formed by its surrounding atoms. And the location of the potential well bottom changes with the instantaneous motions, including the fast and slow motions of the surrounding atoms. So the trajectory of the bottom potential well always varies with time. But, if every atom loses its heat, it will move in its local potential well bottom, named Cold Potential Well Bottom (CPWB), and locate at a state of static equilibrium under a specific external condition. The change of the CPWB's location due to the external condition may be efficient to approximate the atomic slow motion except that the atom shifts from one valley to the other.

At time $t=\tau-\Delta \tau / 2$, the MD instantaneous configuration of atoms is taken to be the initial condition and the CG method is employed to determine the location of CPWB, denoted as $x_{i \alpha}^{\mathrm{CPWB}}(\tau-\Delta \tau / 2)$, under the corresponding external restriction. After a time interval of $\Delta \tau$, the CG method is used again to determine $x_{i \alpha}^{\mathrm{CPWB}}(\tau+\Delta \tau / 2)$. So we use the following equation to approximate the slow velocity of atom $i$ in the $\alpha$-axis direction

$$
v_{i \alpha}^{\mathrm{L}}(\tau) \approx \frac{1}{\Delta \tau}\left(x_{i \alpha}^{\mathrm{CPWB}}(\tau+\Delta \tau / 2)-x_{i \alpha}^{\mathrm{CPWB}}(\tau-\Delta \tau / 2)\right) .
$$

If the atom shifts from one valley of the potential well to the other (i.e. the atom loses stability), the above approximation will be not valid. To estimate the validity of this approximation, we define

$$
v_{i \alpha}^{\mathrm{L}^{*}}(\tau)=\frac{1}{N_{i}^{*}+1}\left(v_{i \alpha}^{\mathrm{L}}(\tau-\Delta \tau)+\sum_{j \in C_{i}^{*}} v_{j \alpha}^{\mathrm{L}}(\tau)\right),
$$

where $C_{i}^{*}$ is a set of the surrounding atoms with satisfying a condition of

$$
\left|v_{i \alpha}^{\mathrm{L}}(\tau)-v_{i \alpha}^{\mathrm{L}}(\tau-\Delta \tau)\right| \leq c_{1}\left|v_{i \alpha}^{\mathrm{L}}(\tau-\Delta \tau)\right|,
$$

in which $0<c_{1}<1$, and $N_{i}^{*}$ is the number of the atoms in $C_{i}^{*}$ with a cut-off distance. If the result of Eq. (3) is close to that of Eq. (2), we take Eq. (2) to approximate the atomic slow velocity; else, we take Eq. (3). A corresponding condition is defined as

$$
\left|v_{i \alpha}^{\mathrm{L}}(\tau)-v_{i \alpha}^{\mathrm{L} *}(\tau)\right| \leq c_{2}\left|v_{i \alpha}^{\mathrm{L} *}(\tau)\right|
$$

to measure how close of the results of Eqs. (2) and (3), where $0<c_{2}<1$. In this paper we take coefficients $c_{1}$ and $c_{2}$ both to be 0.2 .

So, we present a two-step approximate method to determine the atomic slow motion. The first step is based on the change of the location of the cold potential well bottom and the second step is based on the average of the appropriate slow velocities of the surrounding atoms. With this approximate method, the fast and slow motions are split. 


\section{The Numerical Experiments}

The simple tensions of one-dimensional atoms and two-dimensional atoms were performed with the full MD simulations [5]. The CG method [7] was employed to determine the corresponding location of CPWB. For convenience, the atoms are assumed to have the same mass $m$ and interact via the 6-12 Lennard-Jones potential

$$
\phi\left(r_{i j}\right)=4 \varepsilon\left[\left(\sigma / r_{i j}\right)^{12}-\left(\sigma / r_{i j}\right)^{6}\right]
$$

with the characteristic length $\sigma$ and energy $\varepsilon$, in which $r_{i j}$ is the distance from atom $i$ to atom $j$. The potential of atom $i$ is defined as

$$
\Phi_{i}=\frac{1}{2} \sum_{j \in C_{i}} \phi\left(r_{i j}\right),
$$

where $C_{i}$ is a set of its surrounding atoms with a cut-off distance $r_{\mathrm{c}}$. In this paper, the cut-off distance is taken as $3 \sigma$.

For the one-dimensional case, three atoms initially lie along the $x$-axis with a uniform gap of $2^{1 / 6} \sigma$. The leftmost atom denoted as No. 1 is fixed and the rightmost one denoted as No. 3 has a constant velocity rightwards. An initial velocity is given to the No. 2 atom. The Newton's equations of motion are numerically integrated using the velocityVerlet algorithm and the time step is taken to be $0.005 \tau_{\mathrm{LJ}}$, where $\tau_{\mathrm{LJ}}=\sigma(\mathrm{m} / \varepsilon)^{1 / 2}$ denotes the characteristic period of atomic oscillation. After each MD step, the CG method is employed to determine the corresponding location of CPWB.

For the two-dimensional case, there are 333 atoms with the same mass $m$ in a hexagonally packed lattice as shown in Figure 1. The initial dimensions of the sample are $32.748 \sigma \times 10.010 \sigma$. The left three layers of atoms are fixed and the right three layers of atoms have a constant velocity $0.001 v_{\mathrm{LJ}}$ rightwards, where $v_{\mathrm{LJ}}=(\varepsilon / m)^{1 / 2}$. The number of free atoms is 275 and the initial velocities of these atoms are assigned to follow the Maxwell distribution with the mean kinetic energy of $0.05 \varepsilon$. After every 20000 MD steps, the CG method is employed to determine the corresponding location of CPWB.

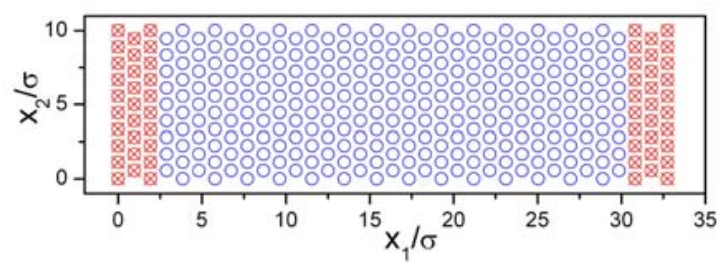

FIGURE 1. The two-dimensional hexagonally packed lattice.

\section{Results and Discussion}

For the simple tension of one-dimensional atoms, the trajectories of the No. 2 atom and the corresponding locations of the CPWB are plotted respectively in Figure 2. The constant velocity of the No. 3 atom was taken as $0.01 v_{\mathrm{LJ}}$ with $v_{\mathrm{LJ}}=(\varepsilon / m)^{1 / 2}$. Two different initial velocities $\pm 0.3 v_{\mathrm{LJ}}$ for the No.2 atom were considered. Figure 2 shows that the trajectories of CPWB remain smooth except a few points and seem to represent the equilibrium-like motions of atom properly. The different initial velocities of the No. 2 atom lead to its different thermal motions and the different thermal motions make it losing the stability along the different paths. It is difficult to predict the losing stability path without a full simulation. The relation between the potential energy of No. 2 atom and its location is illustrated in Figure 3, and so does the relation between the cold potential energy of No. 2 atom and its corresponding location of the CPWB. At the initial stage of loading, the CPW has a single bottom only and the atom vibrates around this bottom. In this state, the deformation of the atomic lattice remains almost uniform. However, with increasing deformation, a peak will emerge from the CPW bottom and the CPW evolves to a double valley. Then the atom may move from one valley to another over the peak. If the atom locates near the peak and the corresponding energy barrier is not high, the CG method without distinguishing the local minimum from maximum may lead to the peak state. With the higher peak, the atom will have to vibrate around one of the two valleys only and the CG method will present the corresponding CPWB. If the motion of the CPW bottom is taken to be the slow motion of the atom approximately, then we can inversely determine the slow velocity of atom from the trajectory of the CPWB using Eq.(2). The results are shown in Figure 4. Before the losing stability, the atom has a stable slow velocity which is 
about a half of the loading velocity. For the case of $v_{2}(0)=0.3 v_{\mathrm{LJ}}$, the atomic slow velocity approaches 0 after the losing stability, which means that the bond of the No. 2 and No. 3 atoms is broken and the No.2 stays with the No. 1 atom. For the case of $v_{2}(0)=-0.3 v_{\mathrm{LJ}}$, the atomic slow velocity approaches $0.01 v_{\mathrm{LJ}}$ after the losing stability, which means that the bond of the No. 1 and No. 2 atoms is broken and the No.2 atom goes with the No. 3 atom. Apparently, the change of the CPWB's location due to the external condition is efficient to approximate the atomic slow motion except that the atom shifts from one valley to the other.

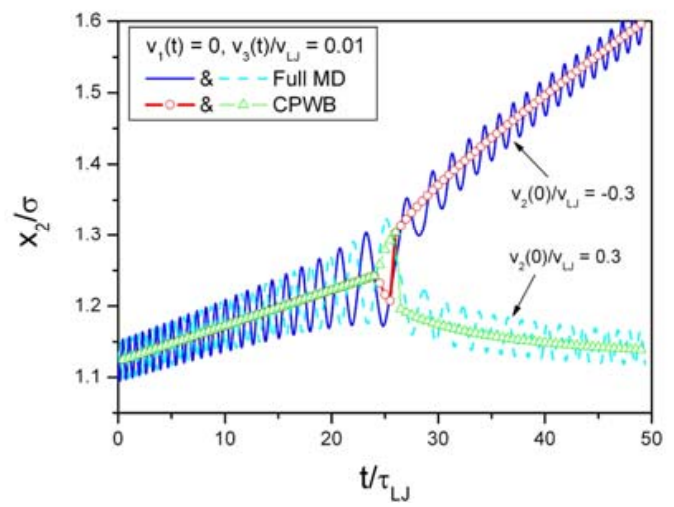

FIGURE 2. The locations of the No.2 atom and the CPW bottom vary with the time.

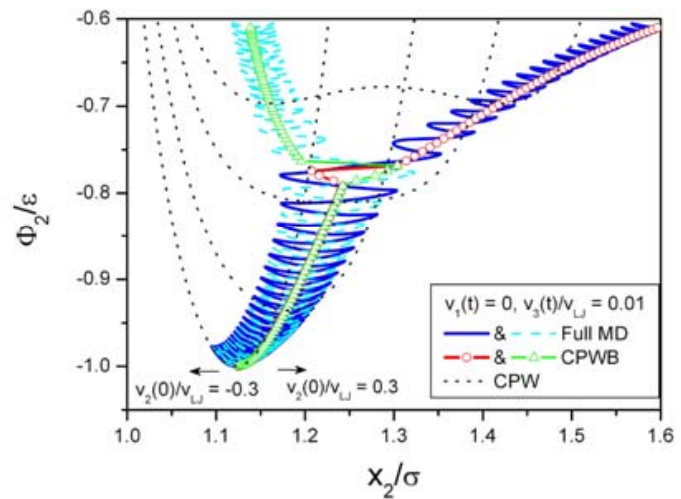

FIGURE 3. The potential energy of the No.2 atom varies with its location and the cold potential energy changes with the location of the CPW bottom.

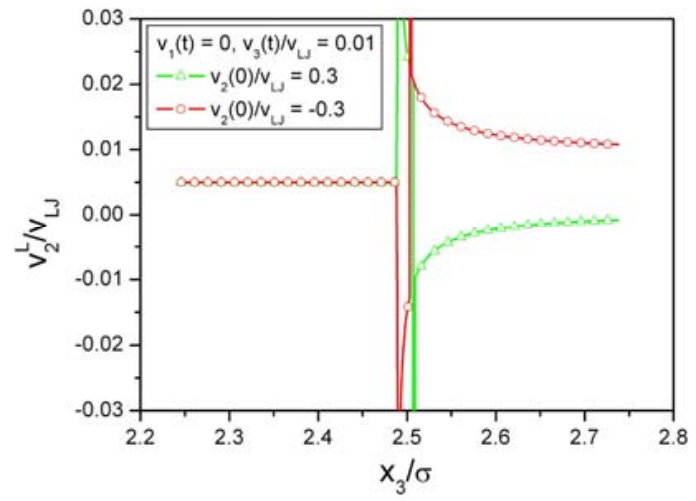

FIGURE 4. The slow velocity of No. 2 atom versus the location of No. 3 atom. 
For the simple tension of two-dimensional atoms, the slow velocity and the time-averaged velocity of the middlemost atom in the $x_{1}$ direction are calculated. The first and second approximations of the slow velocity versus the strain $\varepsilon_{x}$ are shown in Figure 5. For most cases, the first approximation of the slow velocity calculated by Eq. (2) is consistent with that of the physical instinct. But at some strains, the atom loses stability and the first approximation of the slow velocity is not appropriate. After the second-step approximation, the slow velocity seems to be acceptable. In further research, this splitting method will be employed to develop new molecular modeling methods and simulations pertinent to realistic loading conditions of materials. The time-averaged velocity of the middlemost atom in the $x_{1}$ direction is shown in Figure 6 , in which the time-averaged velocity is calculated by

$$
\bar{v}_{i \alpha}(\tau ; \Delta \tau)=\frac{1}{\Delta \tau} \int_{\tau-\Delta \tau / 2}^{\tau+\Delta \tau / 2} v_{i \alpha}(t) d t
$$

and $\Delta \tau$ is taken to be $2 \times 10^{4} \delta t=100 \tau_{\mathrm{LJ}}$. Figure 6 shows that the time-averaged velocity fluctuates with the strain. This is because of that the time interval is not enough large. The evidence is shown below. The integral at the right side of Eq. (8) can be calculated and then we have

$$
\bar{v}_{i \alpha}(\tau ; \Delta \tau)=\frac{1}{\Delta \tau}\left(u_{i \alpha}(\tau+\Delta \tau / 2)-u_{i \alpha}(\tau-\Delta \tau / 2)\right)
$$

where $u_{i \alpha}$ is the displacement. In form, the atomic displacement can be split into the fast and slow components

$$
u_{i \alpha}(t)=u_{i \alpha}^{\mathrm{H}}(t)+u_{i \alpha}^{\mathrm{L}}(t)
$$

and the atomic slow velocity can be written as

$$
v_{i \alpha}^{\mathrm{L}}(\tau)=\frac{1}{\Delta \tau}\left(u_{i \alpha}^{\mathrm{L}}(\tau+\Delta \tau / 2)-u_{i \alpha}^{\mathrm{L}}(\tau-\Delta \tau / 2)\right) .
$$

So the atomic time-averaged velocity becomes

$$
\bar{v}_{i \alpha}(\tau ; \Delta \tau)=v_{i \alpha}^{\mathrm{L}}(\tau)+\frac{1}{\Delta \tau}\left(u_{i \alpha}^{\mathrm{H}}(\tau+\Delta \tau / 2)-u_{i \alpha}^{\mathrm{H}}(\tau-\Delta \tau / 2)\right)
$$

If the last term of the above equation is sufficiently small, the atomic time-averaged velocity will approximately equal the atomic slow velocity. The thermal displacement is comparable with the lattice constant $a_{0}$

$$
\left|u_{i \alpha}^{\mathrm{H}}(\tau+\Delta \tau / 2)-u_{i \alpha}^{\mathrm{H}}(\tau-\Delta \tau / 2)\right|_{\max } \sim a_{0} \sim \sigma
$$

Taking the time interval $\Delta \tau=2 \times 10^{4} \delta t=100 \tau_{\mathrm{LJ}}$, we have

$$
\frac{1}{\Delta \tau}\left|u_{i \alpha}^{\mathrm{H}}(\tau+\Delta \tau / 2)-u_{i \alpha}^{\mathrm{H}}(\tau-\Delta \tau / 2)\right|_{\max } \sim 0.01 v_{\mathrm{LJ}} .
$$

To guarantee the validity of the approximation of the atomic slow velocity by the time-averaged velocity calculated every $20000 \mathrm{MD}$ steps, the condition of $\left|v_{i \alpha}^{\mathrm{L}}(\tau)\right|>>0.01 v_{\mathrm{LJ}}$ should be satisfied. In the above example, the slow velocity is about $0.0005 v_{\mathrm{LJ}}$, so the time-averaged velocity can't be used to approximate the atomic slow velocity.

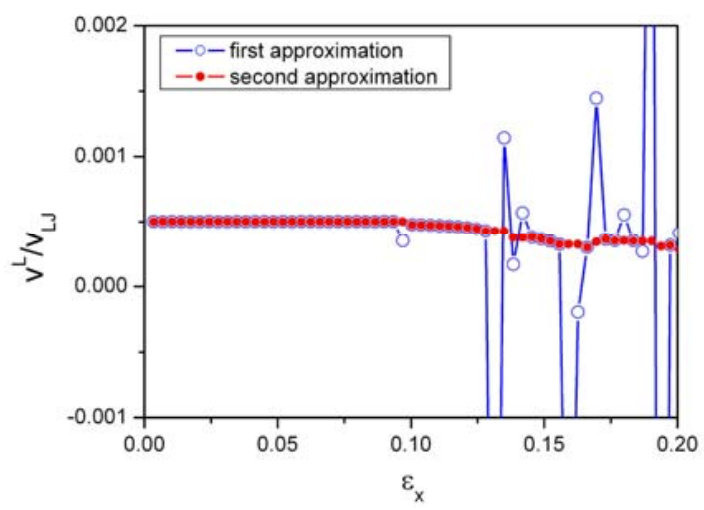

FIGURE 5. The first and second approximations of the slow velocity of the middlemost atom in the $x_{1}$ direction versus the strain. 


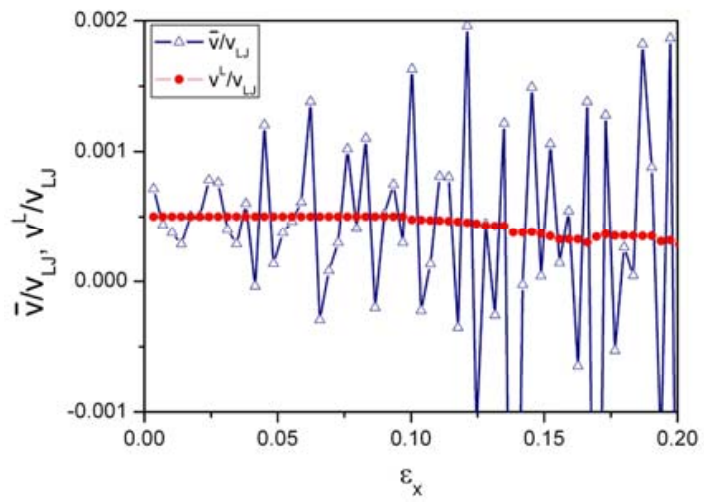

FIGURE 6. Comparison of the time-averaged and slow velocities.

\section{CONCLUSIONS}

A two-step approximate method was presented in this paper to determine the atomic slow motion. The first step is based on the change of the CPWB's location. Most atoms will achieve their appropriate slow velocities in this approximation, but the others should encounter the second step, which is based on the average of the appropriate slow velocities of the surrounding atoms. The simple tensions of one-dimensional atoms and two-dimensional atoms were performed with the full MD simulations. The CG method was employed to determine the corresponding location of CPWB. Results show that our two-step approximate method is appropriate to determine the atomic slow motion under the low strain rate loading. So the atomic motion can be split into the fast and slow components. With the understanding of the coupling mechanisms of molecular thermal motions and mechanical deformation of materials, more efficient molecular modeling methods and simulations pertinent to realistic loading conditions of materials may be explored.

\section{ACKNOWLEDGMENTS}

The contents presented in this paper were supported by the National Natural Science Foundation of China (Nos. 10721202, 10432050, 10772012 and 10732090) and the CAS innovation program (KJCX2-YW-M04).

\section{REFERENCES}

1. W. B. Streett, D. J. Tildesley and G. Saville, Molecular Physics 35, 639-648 (1978).

2. H. Grubmüller, H. Heller, A. Windemuth and K. Schulten, Molecular Simulation 6, 121-142 (1991).

3. M. Tuckerman, B. J. Berne and G. J. Martyna, The Journal of Chemical Physics 97, 1990-2001 (1992).

4. Q. Ma and J. A. Izaguirre, Multiscale Modeling and Simulation 2, 1-21 (2004).

5. D. C. Rapaport, The Art of Molecular Dynamics Simulation, Cambridge: Cambridge University Press, 2004.

6. E. Cances, F. Castella, P. Chartier, E. Faou, C.L. Bris, F. Legoll and G. Turinici, Numerische Mathematik 100, 211232(2005).

7. R. Fletcher and C. M. Reeves, The Computer Journal 7, 149-154 (1964). 